\title{
A New Method of Burgers Vector Identification for Grain Boundary Dislocations from Electron Microscopic Images
}

\author{
By Kenzaburo Marukawa* and Yuji Matsubara**
}

\begin{abstract}
Characteristics of electron microscopic images of dislocations which lie in an inclined grain boundary are examined by theoretical computation. It is assumed that one side of the boundary is in a good diffracting condition and the other is not. The symmetry of the dislocation image varies with the relative values of $\boldsymbol{g} \cdot \boldsymbol{b}$ and $\boldsymbol{g} \cdot(\boldsymbol{b} \times \boldsymbol{u})$, where $\boldsymbol{g}, \boldsymbol{b}$ and $\boldsymbol{u}$ are the reflection vector, the Burgers vector, and the dislocation line vector, respectively. When $\boldsymbol{g} \cdot \boldsymbol{b}$ is large, the image is asymmetric across the dislocation line and the nature of asymmetry depends upon the sign of $\boldsymbol{g} \cdot \boldsymbol{b}$. When $\boldsymbol{g} \cdot(\boldsymbol{b} \times \boldsymbol{u})$ is large, the image is symmetric and it takes light or dark appearance depending upon the sign of $\boldsymbol{g}$. $(\boldsymbol{b} \times \boldsymbol{u})$. When the orientation of the crystal is deviated from the exact diffracting condition, symmetrical or asymmetrical bend of thickness fringes arises in the vicinity of the dislocation. The shape of fringes is also related to the values of $\boldsymbol{g} \cdot \boldsymbol{b}$ and $\boldsymbol{g} \cdot(\boldsymbol{b} \times \boldsymbol{u})$.

It is shown that the Burgers vector can be identified from the knowledge of signs of $\boldsymbol{g} \cdot \boldsymbol{b}$ or $\boldsymbol{g} \cdot(\boldsymbol{b} \times$ $\boldsymbol{u}$ ) for several reflections. This method of identification is compared with others. Especially, it is pointed out that the conventional method of Burgers vector identification cannot be applied to boundary dislocations when only one side of the boundary is in a good diffracting condition. An edge dislocation in a boundary in such a case usually shows quite strong contrast in the micrograph even when $\boldsymbol{g} \cdot \boldsymbol{b}=\mathbf{0}$. A simple reasoning of the strong contrast is given.
\end{abstract}

(Received July 2, 1979)

\section{Introduction}

The identification of dislocations which lie in a grain boundary is much more difficult than in the case of ordinary (not lying in a boundary) dislocations $^{(1) \sim(4)}$. In view of the importance of their studies in relation to the properties of grain boundaries, it was considered worthwhile to develop a reliable and convenient means of identifying them. Essentially two methods have been known for the identification so far; one method makes use of the vanishing criterion of the dislocation image $\boldsymbol{g} \cdot \boldsymbol{b}=0$, and the other is based on matching of the dislocation images with the computed ones ${ }^{(5)(6)}$. Although the former is most convenient for ordinary dislocations, it is not so for boundary dislocations. It is because the vanishing criterion does not neces-

* Department of Applied Physics, Faculty of Engineering, Hokkaido University, Sapporo 060, Japan.

** Graduate School of Engineering, Hokkaido University, Sapporo 060, Japan. Present address: Nippon Electric Co., Ltd., Tokyo 108, Japan. sarily hold for boundary dislocations in the case that one crystal separated by the boundary is in a good diffracting condition and the other is not. As shown theoretically in the following and also experimentally in a later publication, the image of an edge dislocation in such a case usually shows quite strong contrast even when $\boldsymbol{g} \cdot \boldsymbol{b}=0$. The method based on image-matching has been applied to analyse boundary dislocations in several works ${ }^{(1) \sim(3)}$. By this method we can fully utilizes characteristics of dislocation images, but the method is not so convenient because it necessarily requires computation by a high speed computer. Recently, another method has been developed for the identification of ordinary dislocations by one of the present authors ${ }^{(7)}$. This method also makes use of image characteristics, but does not require any computation on each occasion of the identification. In the present paper, the new method is extended for the case of dislocations lying in a boundary.

The new method is based on the examination of the symmetrical natures of dislocation images. Although some characteristics of images

1979 Vol. 20 
of boundary dislocations have been discussed previously ${ }^{(8)}$, no systematic computation of their images has been performed so far. In the following we first examine characteristics of their images by theoretical computation, and relate them to the sign of $\boldsymbol{g} \cdot \boldsymbol{b}$ or $\boldsymbol{g} \cdot(\boldsymbol{b} \times \boldsymbol{u})$, where $\boldsymbol{g}, \boldsymbol{b}$, and $\boldsymbol{u}$ are the reflection vector, the Burgers vector, and the dislocation line vector, respectively. Then we describe how to identify the Burgers vector from the knowledge of these signs. The validity of the new method will be examined later in actual examples.

\section{Computational Procedure}

The geometry of the present computation is shown in Fig. 1. A dislocation lies in an inclined grain boundary and runs from the point B on the bottom (or exit) surface to the point $D$ on the top (or entrance) surface. For simplicity the dislocation is assumed to be perpendicular to the intersection between the boundary plane and the foil surface. We assume that the upper crystal (Crystal 1) is in a good diffracting condition and the lower (Crystal 2) is not diffracting any beam strongly. It should be noted that in this situation only a half of the strain field around the dislocation contributes to the image formation.

Dislocation images can be computed fairly well with the two-beam dynamical theory of electron diffraction by assuming the column approximation. The amplitudes of the primary

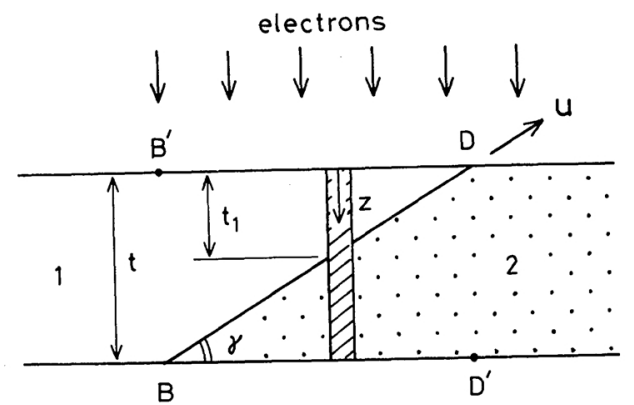

Fig. 1 A side view of a foil containing a grain boundary. Crystal 1 is in a good diffracting condition, and crystal 2 is not. A dislocation lies in the boundary along the direction $\boldsymbol{u}$. Image intensity is computed by integrating the Howie-Whelan equation along the column. The foil thickness is $t$, and the thickness of the diffracting crystal is $t_{1}$. and reflected waves, $T$ and $S$, in the diffracting crystal are given by the Howie-Whelan equation $^{(9)(10)}$ :

$$
\begin{aligned}
& \frac{d T}{d Z}=-R T+(i-Q) S \\
& \frac{d S}{d Z}=(i-Q) T+\left(-R+2 i w+i \frac{d \alpha}{d Z}\right) S,
\end{aligned}
$$

where $R$ and $Q$ are the normal and anomalous absorption coefficient, respectively. The reduced coordinate $Z=\pi z / \xi$ is used, where $z$ is the coordinate measured from the entrance surface along the beam direction and $\xi$ is the extinction distance for the operative reflection. The dimensionless deviation from the Bragg condition is given by $w=|g| \xi \Delta \theta$, where $\Delta \theta$ is the deviation angle of the incident beam. For a positive value of $\Delta \theta$ the second order reflection $\boldsymbol{g}^{\prime}=2 \boldsymbol{g}$ approaches its diffracting condition. For a straight dislocation in an isotropic crystal with the Poisson's ratio $v$, the phase angle $\alpha$ is given by

$$
\begin{aligned}
\alpha= & 2 \pi \boldsymbol{g} \cdot \boldsymbol{R} \\
= & \boldsymbol{g} \cdot \boldsymbol{b}\left(\phi-\phi_{0}\right)+\boldsymbol{g} \cdot \boldsymbol{b}_{\boldsymbol{e}} \frac{\sin 2\left(\phi-\phi_{0}\right)}{4(1-v)} \\
& +\boldsymbol{g} \cdot(\boldsymbol{b} \times \boldsymbol{u})\left[\frac{1-2 v}{2(1-v)} \ln r+\frac{\cos 2\left(\phi-\phi_{0}\right)}{4(1-v)}\right],
\end{aligned}
$$

where $\boldsymbol{R}$ is the lattice displacement produced by the introduction of the dislocation, and $r$ and $\phi$ are cylindrical coordinates with the axis at the dislocation line. The angle $\phi_{0}$ designates the inclination of the slip plane, and $\boldsymbol{b}_{\boldsymbol{e}}$ is the edge component of the Burgers vector. The sign convention for the Burgers vector in this equation is such that $\boldsymbol{b}$ is parallel to $\boldsymbol{u}$ for a right hand screw dislocation and anti-parallel for a left hand screw. In this convention the vector $\boldsymbol{u} \times \boldsymbol{b}$ for an edge dislocation points towards the side where the extra half plane lies.

For the numerical integration of these equations the rapid method developed by Head $^{(5)}$ was followed. The results were presented as pictures drawn by a computer line printer. The Poisson's ratio $v=0.3$ was used. The absorption coefficients $R=Q=0.08$ was assumed. The intensity was normalized to that 
for a perfect crystal. The inclination angle of the boundary plane was taken to be $\gamma=30^{\circ}$. This value is not important in the discussion of image characteristics although it affects the image width. The foil thickness was taken to be $4 \xi$. The foil normal was assumed to be parallel to the beam direction.

If we take the lower crystal as a simple absorbing matter, we obtain too dark contrast in the superposed region and in the nondiffracting crystal compared with experimental images. This discrepancy may arises from the neglect of innelastically scattered electrons, which is known to contribute to the background brightness $^{(11)}$. In order to correct this, the absorption coefficient in the non-diffracting crystal was taken as an adjustable parameter. Its value was adjusted so that the transmitted beam intensity in the non-diffracting crystal was the same as that in a diffracting perfect crystal. Therefore, the level of the background intensity is constant across the boundary. Although this choice of the value was made only for the sake of convenience, it may not affect the important characteristics (e.g. symmetry) of dislocation images.

\section{Characteristics of Dislocation Images}

Computed images of a pure screw dislocation which lies in a boundary are shown in Fig. 2. We are supposed to look down at the pictures from the electron-gun side. Thickness fringes, which are parallel to the intersection between the foil surface and the boundary, diminish their contrast as the thickness $t_{1}$ increases. The contrast in the vicinity of the dislocation line is asymmetric, especially in the thick region ('thick' in the sense that the diffracting crystal is thick). This asymmetry can be related to the sign of $\boldsymbol{g} \cdot \boldsymbol{b}$. Note that for a screw dislocation only the first term in eq. (2) makes a contribution to the image. When we look at the image in the direction of the vector $\boldsymbol{u}$, dark contrast appears to the left of the dislocation and light contrast to the right, irrespective of the value of $w$, if $\boldsymbol{g} \cdot \boldsymbol{b}>0$. If we change the sign of $\boldsymbol{g}$ or $\boldsymbol{b}$, the image is symmetrically reflected across the dislocation line, so that the dark contrast appears to the right. In other words, the dark contrast appears consistently on the side where $d(\boldsymbol{g} \cdot \boldsymbol{R}) / d z<0$. When $w \neq 0$, thickness fringes are bent near at the dislocation. The amount of the bend increases with $|w|$, and the sense of the bend depends on the sign of $w$ as well as the sign of $\boldsymbol{g} \cdot \boldsymbol{b}$ (see Appendix). Since observations are usually made under the condition $w>0$, only the bend under this condition is described. The fringes are then bent towards the thick edge of the diffracting crystal on the left side of the dislocation and towards the thin edge on the right side if $\boldsymbol{g} \cdot \boldsymbol{b}>0$. If the sign of $\boldsymbol{g}$ or $\boldsymbol{b}$ is changed, the sense of the bend is reversed on the both sides.

Figure 3 shows computed images of an edge dislocation. The orientation of its Burgers vector is variously changed. The image in the case $\boldsymbol{g} \cdot(\boldsymbol{b} \times \boldsymbol{u})=0$ is quite similar to that of a screw dislocation except its width. This indicates that the second term in eq. (2) makes a similar contribution to the image as the first.
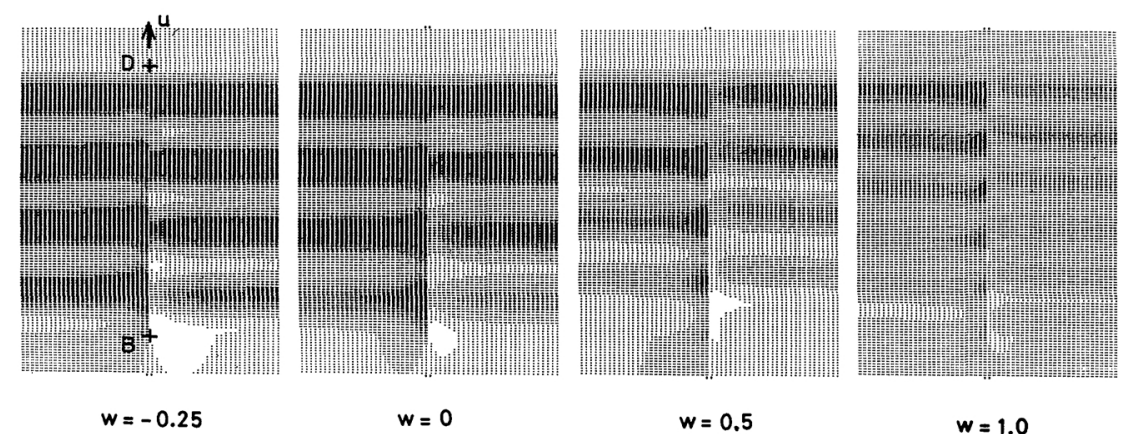

Fig. 2 Computed images of a screw dislocation, which lies in the inclined boundary shown in Fig. 1. The parameters used are; $\boldsymbol{g} \cdot \boldsymbol{b}=1, t=4 \xi, Q=0.08$. The deviation parameter $w$ from the Bragg condition is variously changed. 

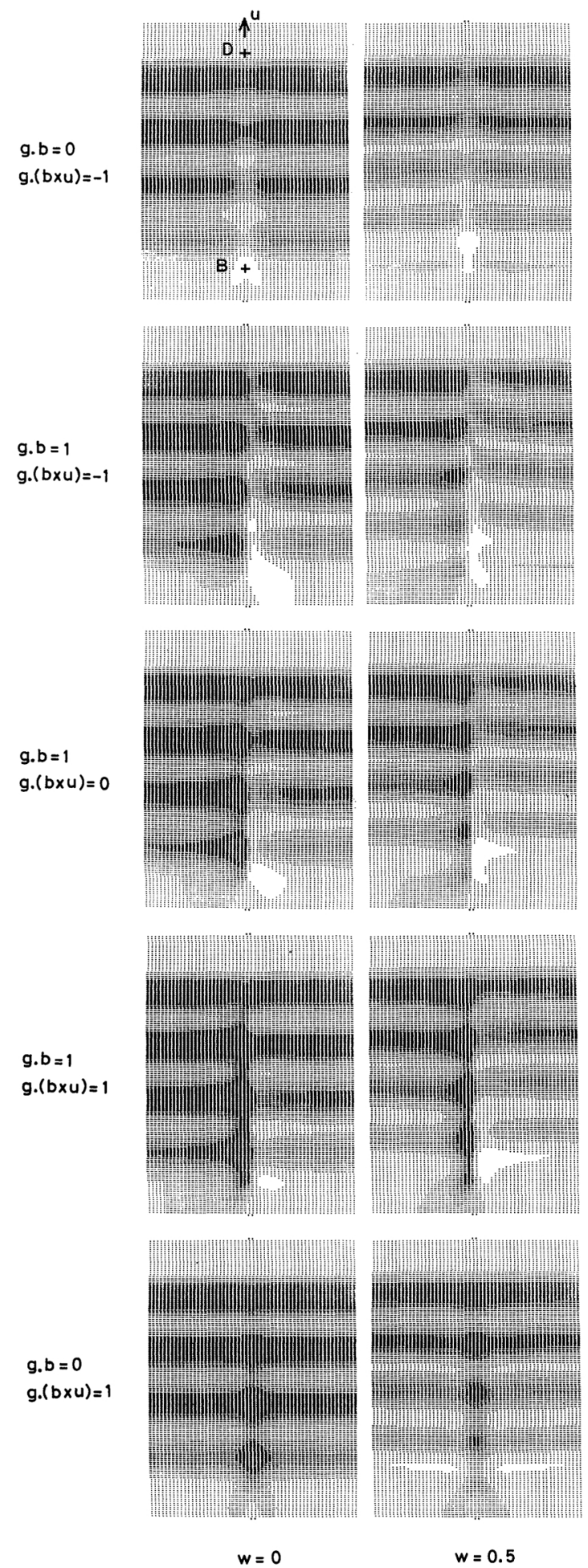

Fig. 3 Computed images of an edge dislocation. The reflection vector is taken to be perpendicular to the dislocation line. $t=4 \xi, Q=0.08$. (a)

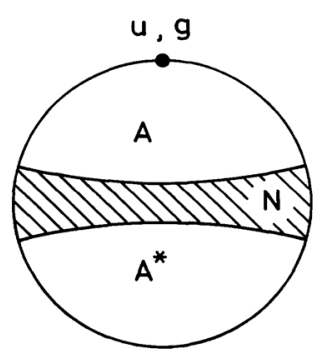

(b)

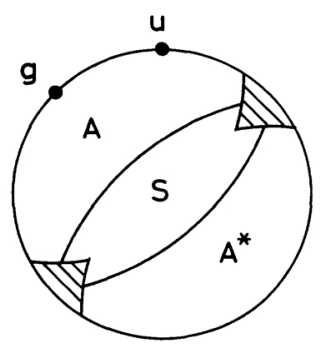

(c)

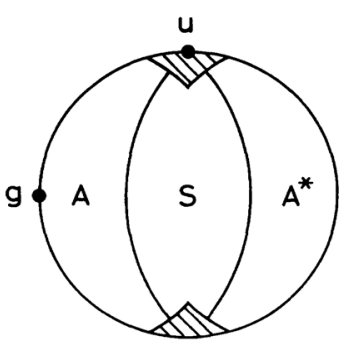

Fig. 4 Stereographic representation of the dependence of the image feature upon the orientation of the Burgers vector. The reflection vector $g$ and the dislocation line vector $\boldsymbol{u}$ are fixed in each figure. An asymmetrical image appears for the Burgers vector in the area $\mathrm{A}$ or $\mathrm{A}^{*}$. Images in the areas $\mathrm{A}$ and $\mathrm{A}^{*}$ are different from each other in the nature of asymmetry. Images in the areas $\mathbf{S}$ and $\mathbf{S}^{*}$, where $\mathbf{S}^{*}$ is on the opposite surface of the stereographic sphere, are roughly symmetrical across the dislocation line, and take a dark or light appearance (see Table 1). The image disappears in the areas $\mathbf{N}$ (the shaded areas).

When $\boldsymbol{g} \cdot \boldsymbol{b}=\mathbf{0}$, the image is symmetric across the dislocation line. The image contrast in this case depends on the sign of $\boldsymbol{g} \cdot(\boldsymbol{b} \times \boldsymbol{u})$. If $\boldsymbol{g} \cdot(\boldsymbol{b} \times$ $\boldsymbol{u})>0$, the contrast is dark. Note that then $d(\boldsymbol{g} \cdot \boldsymbol{R}) / d z<0$ in the diffracting crystal. If $\boldsymbol{g} \cdot(\boldsymbol{b} \times$ $u)<0$, the contrast is light. Also in this case the bend of thickness fringes can be seen when $w \neq 0$. If $\boldsymbol{g} \cdot(\boldsymbol{b} \times \boldsymbol{u})>0$ and $w>0$, the fringes are bent towards the thick edge on the both sides of the dislocation line. If the sign of $\boldsymbol{g} \cdot(\boldsymbol{b} \times \boldsymbol{u})$ is changed, the sense of the bend is reversed.

Image features for all cases, including mixed 
Table 1 Relation between the sign of $\boldsymbol{g} \cdot \boldsymbol{b}$ or $\boldsymbol{g} \cdot(\boldsymbol{b} \times \boldsymbol{u})$ and image characteristics. The area symbols are the same as those in Fig. 4. It is assumed that the upper crystal in the superposed region is in a good diffracting condition and the lower is not. If conversely the lower is in the condition, the tabulated image characteristics for the areas $\mathbf{S}$ and $\mathrm{S}^{*}$ should be interchanged with each other, while those for the areas $A$ and $A^{*}$ remain unchanged. The sense of bend of thickness fringes depends on the sign of $w$.

\begin{tabular}{|c|c|c|c|}
\hline Area & Sign & Contrast & Bend of thickness fringes $(w>0)$ \\
\hline A & $\boldsymbol{g} \cdot \boldsymbol{b}>0$ & $\begin{array}{l}\text { Dark/Light } \\
\text { (left) (right) }\end{array}$ & $\begin{array}{l}\text { To the thick edge/To the thin edge } \\
\text { (left) }\end{array}$ \\
\hline$A^{*}$ & $\boldsymbol{g} \cdot \boldsymbol{b}<0$ & $\begin{array}{l}\text { Light/Dark } \\
\text { (left) (right) }\end{array}$ & $\begin{array}{l}\text { To the thin edge/To the thick edge } \\
\text { (left) }\end{array}$ \\
\hline $\mathbf{S}$ & $\boldsymbol{g} \cdot(\boldsymbol{b} \times \boldsymbol{u})>0$ & Dark & To the thick edge \\
\hline $\mathbf{S}^{*}$ & $\boldsymbol{g} \cdot(\boldsymbol{b} \times \boldsymbol{u})<0$ & Light & To the thin edge \\
\hline
\end{tabular}

dislocations, are summarized in Fig. 4, where $|\boldsymbol{g}|=|\boldsymbol{b}|=1$ is assumed and the dependence of the image feature upon the orientation of the Burgers vector is examined. For the Burgers vector in the area $A$ or $A^{*}$, where the term $\boldsymbol{g} \cdot \boldsymbol{b}$ in eq. (2) makes a large contribution, the image is asymmetric across the dislocation line, as shown typically in Fig. 2. For the one in the area $\mathrm{S}$ or $\mathrm{S}^{*}$, where the term $\boldsymbol{g} \cdot(\boldsymbol{b} \times \boldsymbol{u})$ has a large value, the image is more or less symmetric. The image in the area $A^{*}$ is similar to that in the area $A$ except that the nature of asymmetry is reversed. The images in the areas $\mathrm{S}$ and $\mathrm{S}^{*}$ take dark and light appearances, respectively. There are two keys for characterizing the image feature, i.e. the contrast of the dislocation image and the bend of thickness fringes. As these features change with the orientation of the Burgers vector in a similar manner, the same division of areas on the stereographic representation can be used for both. The shape of thickness fringes is discussed in some detail in Appendix. The interrelation between the two features is also pointed out there. The lines deviding the areas were drawn by inspecting computed images for a variety of the Burgers vectors. As the change from an asymmetric image to a symmetric one is gradual, the deviding lines do not bear a serious meaning. The area $\mathrm{N}$, in which the image dissappears, is centered near at the point where the conditions $\boldsymbol{g} \cdot \boldsymbol{b}=\mathbf{0}$ and $\boldsymbol{g} \cdot(\boldsymbol{b} \times \boldsymbol{u})=\mathbf{0}$ are satisfied. In Fig. 4(a) the area $\mathrm{N}$ is defined by the condition $|\boldsymbol{g} \cdot \boldsymbol{b}| \leqq 0.25$.

The image characteristics are summarized in Table 1. With this table and Fig. 4, we can determine the signs of $\boldsymbol{g} \cdot \boldsymbol{b}$ and $\boldsymbol{g} \cdot(\boldsymbol{b} \times \boldsymbol{u})$. It should be noted that in all these results the upper crystal in the superposed region is assumed to be in a good diffracting condition. If the lower is in the condition and the upper is not, the image features in the areas $S$ and $S^{*}$ are changed. More precisely, if the lower is in a good diffracting condition and if $\boldsymbol{g} \cdot(\boldsymbol{b} \times \boldsymbol{u})>0$, the dislocation image takes a light appearance and thickness fringes are bent towards the thin edge of the diffracting crystal for the deviation $w>0$. On the other hand, the nature of asymmetry of images in the areas $A$ and $A^{*}$ is not changed on the same conversiont. In experiments, it is necessary to determine the sense of inclination of the boundary in order to know the diffracting side. For example, in Fig. 1 we have to know which of $\mathrm{BD}$ or $\mathrm{B}^{\prime} \mathrm{D}^{\prime}$ is the boundary plane. Thus, the sign of $\boldsymbol{g} \cdot \boldsymbol{b}$ can be determined solely by examining the image, but the sign of $\boldsymbol{g} \cdot(\boldsymbol{b} \times \boldsymbol{u})$ can be determined only if the sense of inclination of the boundary is known.

\section{Method of the Burgers Vector Identification}

The present method of the Burgers vector identification is similar to that in the case of ordinary dislocations ${ }^{(7)}$. The dislocation of

$\dagger$ The images for the reflection $g$ in the case II (Crystal 2 in Fig. 1 is in a good diffracting condition) are given by the $180^{\circ}$ rotation of the images for the reflection $(-g)$ in the case $I$ (Crystal 1 is in the diffracting condition). This holds irrespective of the orientation of $\boldsymbol{b}$. 


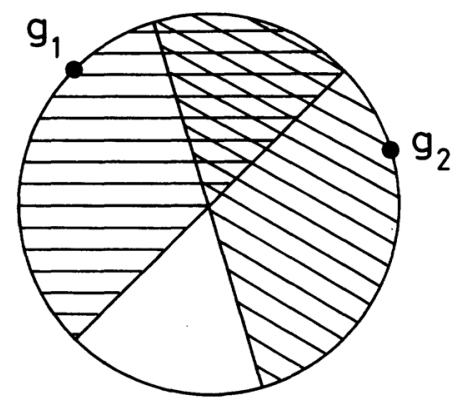

Fig. 5 Illustration of the method for the Burgers vector identification. The possible orientations of the Burgers vector are supposed to distribute in the stereographic sphere. The shaded areas indicate the areas in which $\boldsymbol{g}_{1} \cdot \boldsymbol{b}>\mathbf{0}$ or $\boldsymbol{g}_{2} \cdot \boldsymbol{b}>\mathbf{0}$. If the signs of $g_{1} \cdot \boldsymbol{b}$ and $g_{2} \cdot \boldsymbol{b}$ are known from the image characteristics, say, if both are possitive, only the orientations within the doubly shaded area are allowed for the Burgers vector.

which the Burgers vector is to be determined should be photographed in several different reflections. The first step is to arbitrarily choose the sense of the dislocation line vector. This implicitly fixes the sense of the Burgers vector. Then, we can determine the sign of $\boldsymbol{g} \cdot \boldsymbol{b}$ or $\boldsymbol{g} \cdot(\boldsymbol{b} \times \boldsymbol{u})$ from the image characteristics, as mentioned in the above section. For simplicity of the description, we first neglect the sign of $\boldsymbol{g} \cdot(\boldsymbol{b} \times \boldsymbol{u})$. Figure 5 illustrates how to determine the orientation of the Burgers vector. If the sign of $\boldsymbol{g} \cdot \boldsymbol{b}$ is known for a particular reflection, say $\boldsymbol{g}_{1}$, the possible orientation of $\boldsymbol{b}$ is confined within a hemisphere (i.e. the one with $g_{1}$ as the pole if $\boldsymbol{g}_{1} \cdot \boldsymbol{b}>0$, or the other hemisphere if $\boldsymbol{g}_{1} \cdot \boldsymbol{b}<0$ ). In the same manner, the knowledge of the sign of $\boldsymbol{g} \cdot \boldsymbol{b}$ for another reflection, say $\boldsymbol{g}_{2}$, gives another hemisphere. Then, the possible orientation of $\boldsymbol{b}$ is confined within the smaller area which is the common area of the two hemispheres. Repeating the same procedure for other reflections, we can confine the possible orientation in a smaller and smaller area, and finally choose the Burgers vector uniquely from among the crystallographically allowable vectors. It should be noted that in this selection we have to distinguish between vectors different only in the sign. For example, the number of possible vectors of the type $\langle 100\rangle$ is six.

If the possible orientation of $\boldsymbol{b}$ is confined by a single photograph in a smaller area than a hemisphere, the number of steps required for the unique selection of $\boldsymbol{b}$ is reduced. This is really the case, as shown in Fig. 4. That is, if the knowledge of the sign of $\boldsymbol{g} \cdot(\boldsymbol{b} \times \boldsymbol{u})$ is also available, we can confine the orientation in a smaller area, A, A*, S, S*, or N.

The present method is related to the signs of various variables. As to the sign of $w$ and the sense of inclination of the boundary, remarks are given above in Table 1. If we want to know the sense of the Burgers vector correctly, we have to take into account the $180^{\circ}$ rotation correction of the diffraction pattern, which is occasionally necessary in a particular lens system of an electron microscope. Even when we need not know the sense, the sign of $g$ should be taken into account in a consistent manner (either with or without the rotation correction). Since the sense of the Burgers vector is reversed on the reversal of the vector $\boldsymbol{u}$, the first choice of the sense of $\boldsymbol{u}$ should be retained in the course of the identification procedure.

\section{Discussion}

The image of a boundary dislocation is different in many respects from that of an ordinary dislocation. The most important difference may be the one in the visibility of an edge dislocation in the case $\boldsymbol{g} \cdot \boldsymbol{b}=0$, because it concerns the validity of the conventional method of the Burgers vector identification. As shown in Fig. 3, an edge dislocation in a boundary shows fairly strong contrast even when $\boldsymbol{g} \cdot \boldsymbol{b}=0$. A simple reasoning for the origin of this difference can be made. The image intensity is determined by the strain field of a dislocation through the term $d \alpha / d z$ in eq. (1). Roughly speaking, it is obtained by integrating, as it were, a weightened value of $d \alpha / d z$ along a column. In the case in hand, only the third term in eq. (2) makes a contribution. When we consider the variation of this term along a particular column, we immediately recognize that $d \alpha / d z$ changes its sign at the depth of the dislocation line. Therefore, in the case of an ordinary dislocation, where the upper and the lower parts of the crystal equally diffract the electron beam, the contributions 
from the upper and the lower parts almost compensate with each other, and a weak contrast of the dislocation image results. On the other hand, in the case of a boundary dislocation, only one half, either the upper or the lower, of the strain field contributes to the image, so that a strong dark or light contrast of the dislocation image arises. Computed images have shown that the contrast is dark consistently when $d \alpha / d z<0$.

In general, the identification of a dislocation in a boundary is much more difficult than in the case of an ordinary dislocation. It is partly because the use of the vanishing condition of images is more limited and partly because possible vectors to consider for the choice of Burgers vector are more numerous. Therefore, for the identification we have to make use of information obtained from images as much as possible. The present method of the identification has presented a way of utilizing image characteristics in addition to its visibility.

\section{Acknowledgements}

The authors would like to appreciate Prof. Shin'ichi Sato for encouragement and helpful discussion through the course of this work. The computation of dislocation images was performed at Hokkaido University Computing Center.

\section{REFERENCES}

(1) P. Humble and C. T. Forwood: Phil. Mag., 31 (1975), 1011.

(2) C. T. Forwood and P. Humble: Phil. Mag., 31 (1975), 1025.

(3) Y. Ishida, M. Mori and F. Iida: Acta Met., 25 (1977), 815.

(4) W. A. T. Clark and D. A. Smith: Phil. Mag., A38 (1978), 367.

(5) A. K. Head: Aust. J. Phys., 20 (1967), 557.

(6) A. K. Head, P. Humble, L. M. Clarebrough, A. J. Morton and C. T. Forwood: Computed Electron Micrographs and Defect Identification, North-Holland Pub. Co., Amsterdam, (1973).

(7) K. Marukawa: Phil. Mag., A40 (1979), 303.

(8) R. M. Fisher, H. Hashimoto and J. W. Negele: Sixth International Congress for Electron Microscopy, Maruzen, Tokyo, (1966), p. 79.

(9) A. Howie and M. J. Whelan: Proc. Roy. Soc. Lond., A263 (1961), 217.

(10) P. B. Hirsch, A. Howie, R. B. Nicholson, D. W. Pashley and M. J. Whelan: Electron Microscopy of Thin Crystals, Butterworths, London, (1965).
(11) A. Ichiyama: Japan. J. Appl. Phys., 7 (1968), 1425.

(12) F. W. Schapink: Phys. Status Solidi, (a)29 (1975), 623.

\section{Appendix}

\section{Approximate Theory of the Bend of Thickness Fringes}

We start with another form of the HowieWhelan equation, which is based on the dispersion surface construction ${ }^{(10)}$ In this scheme electrons in a crystal are represented by a linear combination of two Bloch waves, whose wave vectors are slightly different from each other. The difference brings about a beat of the two waves and causes thickness fringes in an wedge-shaped crystal. In a perfect crystal the beat period in thickness is

$$
\xi^{\prime}=\xi /\left(1+w^{2}\right)^{1 / 2} .
$$

If the strain in a crystal produces an additional phase difference between the two waves, the beat period is modified. Then the bend of thickness fringes arises. The amplitudes $\psi^{(1)}$ and $\psi^{(2)}$ of the two waves obey the equations,

$$
\begin{aligned}
& \frac{d \psi^{(1)}}{d z}=\frac{i}{2} \frac{d \alpha}{d z}\left[(1-\cos \beta) \psi^{(1)}\right. \\
& \left.-\sin \beta \exp \left(2 \pi i z / \xi^{\prime}\right) \psi^{(2)}\right], \\
& \frac{d \psi^{(2)}}{d z}=\frac{i}{2} \frac{d \alpha}{d z}\left[(1+\cos \beta) \psi^{(2)}\right. \\
& \left.-\sin \beta \exp \left(-2 \pi i z / \xi^{\prime}\right) \psi^{(1)}\right], \\
& \cos \beta=w /\left(1+w^{2}\right)^{1 / 2},
\end{aligned}
$$

where $\alpha$ is given by eq. (2). The first terms in the parentheses represent the intraband scattering and the second terms represent the interband scattering. When $|w| \gg 1$, the second terms can be neglected. Then the equations can be easily integrated. The results are:

$$
\begin{aligned}
& \psi^{(1)}=\psi_{0}^{(1)} \exp \left[\frac{i}{2}(\alpha(z)-\alpha(0))(1-\cos \beta)\right], \\
& \psi^{(2)}=\psi_{0}^{(2)} \exp \left[\frac{i}{2}(\alpha(z)-\alpha(0))(1+\cos \beta)\right],
\end{aligned}
$$

where $\psi_{0}^{(1)}$ and $\psi_{0}^{(2)}$ are constants and $\alpha(z)$ is the value at the depth $z$. As we can set the two waves in the same phase at the entrance surface, the additional phase difference between the two 
waves at the boundary plane is

$$
\left(\alpha\left(t_{1}\right)-\alpha(0)\right) \cos \beta \approx \pm\left(\alpha\left(t_{1}\right)-\alpha(0)\right) \text {. }
$$

The double sign depends on the sign of $w$.

When $w \approx 0$, the phase difference due to the first terms in eq. (A2) becomes zero, but then the second terms cannot be neglected. Although the analytical solution is not obtained in this case, computed images show that the additional phase difference (i.e. the shift of thickness fringes) is not appreciable unless $\boldsymbol{g} \cdot \boldsymbol{b} \geqq 3$. This suggests that the contribution of the interband scattering to the phase difference may be small. Therefore, the first of eq. (A4) may hold even at a smaller value of $w$. In the following we assume that $w \gg 1$.

As the beat period corresponds to an phase difference of $2 \pi$ the shape of thickness fringes is given by

$$
2 \pi t_{1} / \xi^{\prime}+\alpha\left(t_{1}\right)-\alpha(0)=2 \pi n . \quad(n: \text { integer })
$$

As the shift of the fringe brings about the shift of the column position at which $\alpha$ 's are evaluated, the equation can be solved only by numerical calculation, where $t_{\mathbf{1}}$ is taken as a variable. However, in the condition (d) $\left.d t_{1}\right)\left[\alpha\left(t_{1}\right)-\alpha(0)\right] \ll 2 \pi / \xi^{\prime}$, the amount of the shift can be shown to be proportional to $\Delta \alpha=\alpha\left(t_{1}\right)$ $\alpha(0)$. If $\Delta \alpha>0$, the fringe shift occurs towards the thinner part of the crystal (i.e. the part having a smaller value of $t_{1}$ ). It can be shown that this approximation holds rather well except the first fringe. The value of $\Delta \alpha$ was calculated from eq. (2) as a function of the column position shown in Fig. A1. The results for an edge dislocation are shown in Fig. A2. These curves approximately represent the shape of thickness fringes. The sense of each axis is chosen so as to be fit for Fig. 3. For example when $\phi_{0}=0^{\circ}$, i.e. $\boldsymbol{g} \cdot \boldsymbol{b}>0$, the fringes are bent towards the thin edge $(\Delta \alpha>0)$ on the right side $(y>0)$ of the dislocation line. The first term is eq. (2) produces a discontinuous jump of fringes at the dislocation line, which amounts to $\pi \boldsymbol{g} \cdot \boldsymbol{b}$. If $\boldsymbol{g} \cdot \boldsymbol{b}=2$, fringes are continuously connected to the neighboring fringes.

Similar thickness fringes are observed in a tapering foil. The shape of these can be calculated in a similar way, provided that $t_{1}$ is

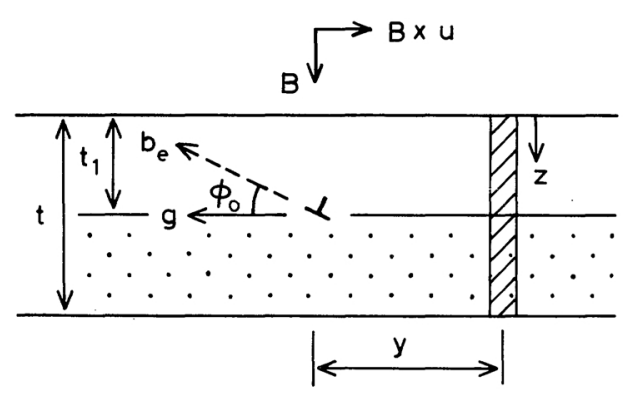

Fig. A1 A cross section, of the same foil as in Fig. 1, which is parallel to the beam direction $B$ and the vector $B \times u$. The vector $\boldsymbol{b}_{e}$ and the angle $\phi_{0}$ are not in the plane of the figure.
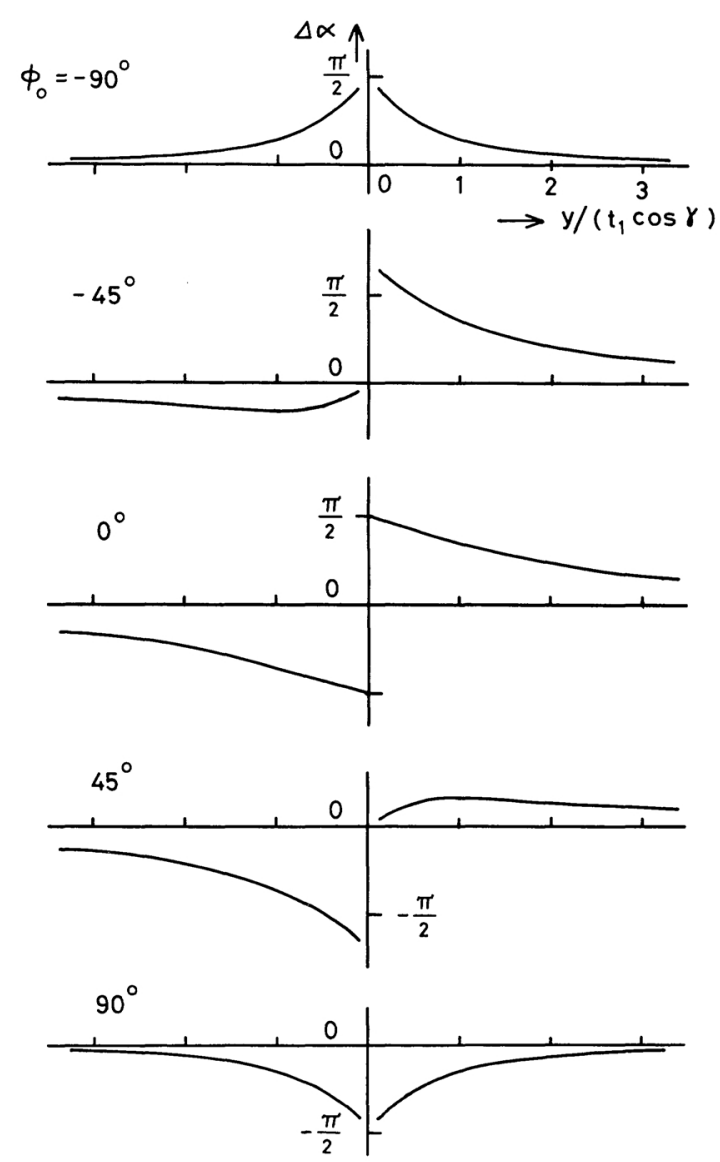

Fig. A2 The phase difference $\Delta \alpha=\alpha\left(t_{1}\right)-\alpha(0)$ as a function of the column position $y$. These curves approximately represent the shape of thickness fringes. An edge dislocation is supposed to lie at $y=0$. The reflection vector is taken to be perpendicular to the dislocation line, as shown in Fig. A1. It is assumed that $|\boldsymbol{g}|=|\boldsymbol{b}|=1$. The angle $\phi_{0}$ denotes the angle between $g$ and $b$. 
replaced by $t$. Schapink ${ }^{(12)}$ has discussed the shape of fringes in this case, although his approach to the problem is seemingly quite different from the present. It is to be noted that the amount of the discontinuous jump of fringes is roughly doubled in this case.

It is interesting to note that the curves fairly well represent also the contrast variation across the dislocation line. In this comparison we must take the ordinate of Fig. A2 as the image intensity which is measured from the back- ground level. Also we must replace the abscissa by $y /(\zeta \cos \gamma)$, where $\zeta$ takes the value $0.3 \xi$ to $0.8 \xi$. For example, the first curve in Fig. A2 indicates light appearance of the dislocation image and the last shows dark appearance. These features accord well with the corresponding computed images in Fig. 3. This correspondence implies that the image contrast is well explained by the phase difference $\Delta \alpha$ which is evaluated in the layer of thickness $\zeta$ adjoining the dislocation. 